\title{
On Chasles' Property of the Helicoid in Tri-Twisted Real Ambient Space
}

\author{
Peter T. Ho, Lucy H. Odom, Bogdan D. Suceavă
}

\begin{abstract}
An elementary property of the helicoid is that at every point of the surface the following condition holds: $\cot \theta=C \cdot d$, where $d$ is the distance between an arbitrary point to the helicoid axis, and $\theta$ is the angle between the normal and the helicoid's axis. This rigidity property was discovered by M. Chasles in the first half of the XIXth century. Starting from this property, we give a characterization of the so-called tri-twisted metrics on the real three dimensional space with the property that a given helicoid satisfies the classical invariance condition. Similar studies can be pursued in other geometric contexts. Our most general result presents a property of surfaces of rotation observing an invariance property suggested by the analogy with Chasles's property.
\end{abstract}

\section{Introduction}

The helicoid was originally brought to light by Meusiner [16], and its important role in the theory of surfaces was recognized early in the development of differential geometry. In 1842, E. Catalan proved that the only ruled surfaces that are minimal are either a part of a plane or a part of a helicoid [3]. Note that in Catalan's original proof the helicoid is described by the equation

$$
y=-C \arctan \frac{x}{z}
$$

Key Words: Helicoid, catenoid, submanifold, helices, twisted metrics, isometric immersion, rotation surface.

2010 Mathematics Subject Classification: Primary 53A05, 53B20; Secondary 51M99, 53A55, 53B25.

Received: September 17, 2013.

Accepted: December 2, 2013. 
D. Hilbert and S. Cohn-Vossen wrote in section 30 of their monograph [13] that "the most general helicoid is the surface swept out by an arbitrary space curve performing a uniform screw motion about a fixed axis." Thus, the idea of a helicoid ruled along an arbitrary curve is quite old. More recently these kinds of surfaces have been called "bent helicoids". Bent helicoids along a generating curve have been studied quite a lot recently. Although we do not aim here to summarize any recent developments, in support to our claim we would like to remind just one direction of study, since bent helicoids along a circle play a central role in [17] as well as in [1]. This idea will motivate our Example 2. Twisting the metric in the ambient space is an idea dual to bending the helicoids, and we'll see this idea materialized in our theorem.

Reading further into D. Hilbert and S. Cohn-Vossen's monograph [13], we see that section 33 is dedicated to the study of bendings leaving a surface invariant. A natural question complementing this discussion is what deformations of the ambient space where the helicoid lies preserve other rigid properties of a specific helicoid. If the question has to do with the geometry of the ambient space, then the outcome is a study in submanifold geometry, closer in spirit to the developments in $[4,5,8]$. This quest, whose origin can be traced in section 33 of Hilbert and Cohn-Vossen monograph is the motivation of our present study. We provide further details in the next section.

Helicoids play an important role in recent developments in the geometry of surfaces. C. J. Costa [9], D. Hoffman and W. H. Meeks [10, 11] have disproved a longstanding conjecture which stated that the only complete embedded minimal surfaces in $\mathbb{R}^{3}$ of finite topological type are the plane, the catenoid, and the helicoid. This conjecture turned out to be false as there exists a family of complete embedded minimal surfaces defined on a genus $k-1 \quad(k>1)$ compact Riemann surface with three points removed. For a discussion of Costa's surface in the context of the geometry of surfaces with constant mean curvature, see [14], p.65 et al. To further pinpoint the important place of the helicoid in the geometry of surfaces in the three-dimensional real space, we remind that D. E. Blair and Th. Koufogiorgios proved that the only ruled surface in Euclidean space with vanishing second Gaussian curvature is a piece of a helicoid [2].

Twisted product metrics have been introduced by B.-Y. Chen in $[6]$ as a generalization of warped product metrics [20].

For the simplicity of notation we denote the Riemannian metric by the classical dot product since our work will be done in $\mathbb{R}^{3}$. However, we'll specify in the context of every theorem what metric we use. 


\section{Motivation for Our Study: Chasles' Angle Invariance Property for Helicoids}

Dirk J. Struik pointed out [23] that Lancret's theorem was originally stated in 1802: A necessary and sufficient condition that a curve be of constant slope is that the ratio of curvature to torsion be constant. However, the first proof was given by B. de Saint Venant in 1845, as mentioned in [23], p.34.

Today, Lancret's theorem appears in several introductory texts (see e.g. Problem 3104, p. 156 in [15]). With all its extended exposure, the geometry of helices still reveals new interesting properties and connections with other areas, as exemplified by B.-Y. Chen's study of rectifying curves [7].

Lancret's theorem depends on the metric in the ambient space. If we deform the metric in the ambient space, the statement of Lancret's theorem does not necessarily hold true. Since a helicoid is a ruled surface generated by the rulings lying along the normal direction of a helix, we'll be looking at the corresponding property in the geometry of surfaces. This fact is usually stated as follows (Problem 4.2.6 [21]). Let $\sigma(u, v)=(v \cos u, v \sin u, \lambda u)$, be the helicoid with pitch $\lambda$, where $\lambda$ is a constant. Then the cotangent of the angle that the standard unit normal of $\sigma$ at a point $p$ makes with the $z$-axis is proportional to the distance of $p$ from the $z$-axis. The proof is just a simple direct computation. This is equivalent to the classical theorem of Chasles [23], p.194: The tangent of the directed angle between a tangent plane at a point $P$ of a generator of a nondevelopable ruled surface and the central plane is proportional to the distance of $P$ to the central point.

We'll say that a ruled surface $\sigma$ has Chasles' angle invariance property if there exists a curve $\gamma(t)$ (called axis of the ruled surface) such that the cotangent of the angle that the standard unit normal of $\sigma$ at a point $p$ makes with $\gamma$ 's tangent vector is proportional to the distance of $p$ from the axis $\gamma(t)$. We note that this curve $\gamma(t)$ plays the role of the general curve described by Hilbert and Cohn-Vossen in section 30 in [13] ( as we mentioned above).

To complete our discussion, we remind here that $\mathrm{O}$. Bonnet focused on another condition on constant angles to characterize the geometry of ruled surfaces. His theorem states the following. If a curve on a ruled surface satisfies any of the three conditions: (a) of being a geodesic, (b) of being a striction line, (c) of intersecting the generators at constant angles, then any two of these three conditions implies the third. (See [23], p.195.)

\section{Study of the Axial Property Through Examples}

A natural question to ask is this: how remarkable is Chasles' angle invariance property? We explore this idea by two examples. First, we look at the standard 
helicoid, but with a different "axis", i.e. we change the curve that supports the rulings. In the second example, we look at a bent helicoid around a planar circle. In each of these examples, we see the relation between $\cot \theta$ and the distance between an arbitrary point to the helicoid's axis.

Example 1. Consider the helix given by $\gamma: \mathbb{R} \rightarrow \mathbb{R}^{3}$, $\gamma(t)=(a \cos t, a \sin t, b t)$. Then the velocity is $\gamma^{\prime}(t)=(-a \sin t, a \cos t, b)$, and the acceleration is $\gamma^{\prime \prime}(t)=(-a \cos t,-a \sin t, 0)$. Define the surface $\sigma$ : $\mathbb{R} \times(-1,1) \rightarrow \mathbb{R}^{3}$ by

$$
\sigma(t, s)=\gamma(t)+s \gamma^{\prime \prime}(t)
$$

Then we end up with the parametrization:

$$
\sigma(t, s)=((1-s) a \cos t,(1-s) a \sin t, b t)
$$

To respond to our question, we compare $\cot \angle\left(N, \gamma^{\prime}\right)$ and the distance between $\sigma(t, s)$ and $\gamma(t)$. We denote this distance by $d$. To simplify further our computations, we take $a=1$. We obtain

$$
\cot \theta=\frac{ \pm b}{1-\frac{\left\|\gamma^{\prime}(t)\right\|}{d}}
$$

In conclusion, we can not talk about the same angle invariance property if the axis of the helicoid is another helix among those that are generating the same ruled surface.

In this sense, the position enjoyed by the $z$-axis is central and the property obtained as a consequence of Lancret's theorem is remarkable.

Example 2. Consider the planar curve $c(u)=(R \cos u, R \sin u, 0)$, for $u \in(0,2 \pi)$. Then consider the helix twisting around inside the torus $k$ times, where $k$ is an even natural number (to insure the existence of the normal, which is needed in Chasles' angle invariance property). Denote by $\gamma(u)$ the position vector that has the coordinate functions:

$$
\begin{gathered}
x(u)=(R+r \cos (k u)) \cos u, \\
y(u)=(R+r \cos (k u)) \sin u, \\
z(u)=r \sin (k u) .
\end{gathered}
$$

We write the parametrization of the bent helicoid in the torus of radii $\mathrm{R}$ and r with $R>r>0$. Note that $r$ is not essential for the geometry of the ruled surface. For $-1<s<1$ : The bent helicoid in the torus is:

$$
\sigma(u, s)=c(u)+s(\gamma(u)-c(u))=(1-s) c(u)+s \gamma(u)
$$


Based on these geometric considerations, we obtain the bent helicoid in the torus around the circle in the $x y$-plane of radius $R$ :

$$
\begin{gathered}
\sigma:(0,2 \pi) \times(-1,1) \rightarrow \mathbb{R}^{3} \\
\sigma(u, s)=((R+s \cos (k u)) \cos u,(R+s \cos (k u)) \sin u, s \sin (k u)) .
\end{gathered}
$$

A short computation shows that the distance between $\sigma(u, s)$ and $c(u)$ in the canonical metric in $\mathbb{R}^{3}$ is $|s|$. A more elaborated direct computation gets for $\theta$, the angle between the normal and $c^{\prime}(u)$ the following expression:

$$
\cot \theta=\frac{s k \sin (k u)[\sin (k u)+\cos (k u)]}{R+s \cos (k u)} .
$$

Note that the denominator of this fraction represents the distance from a moving point to the center of the torus, i.e. the center of the circle described by $c(s)$. In any case, the angle invariance property is not observed in the form stated in the previous section.

\section{Twisted Metrics on $\mathbb{R}^{3}$ in Which Chasles' Angle In- variance Property Is Preserved}

In an attempt to find which minimal conditions guarantee that we obtain a particular geometry, we are performing a type of inquiry that is similar to the axiomatic analysis described by David Hilbert in [12] and discussed more recently by $\mathrm{V}$. Pambuccian in [19] from a logical standpoint. The type of inquiry we perform here aims to find out what are the geometric conditions sufficient to hold in the ambient space so that the condition $\cot \theta=C \cdot d$ holds true. Both terms $\cot \theta$ and the distance $d$ depend on the Riemannian metric in the ambient space.

We prove the following result.

Theorem 4.1. Let $\sigma:(0, \infty) \times(0,2 \pi) \rightarrow \mathbb{R}^{3}, \sigma(u, v)=(k v, u \cos v, u \sin v) \subset$ $\mathbb{R}^{3}$, with $k>0$, be an isometric immersion into the three dimensional real space endowed with the tri-twisted metric given by

$$
d s^{2}=f^{2}\left(x_{1}, x_{2}, x_{3}\right) d x_{1}^{2}+h^{2}\left(x_{1}, x_{2}, x_{3}\right) d x_{2}^{2}+q^{2}\left(x_{1}, x_{2}, x_{3}\right) d x_{3}^{2},
$$

with the $f, h, q \in C^{\infty}\left(\mathbb{R}^{3}\right), f\left(x_{1}, x_{2}, x_{3}\right)>0, h\left(x_{1}, x_{2}, x_{3}\right)>0$, and $q\left(x_{1}, x_{2}, x_{3}\right)>0$. Then the helicoid given by $\sigma(u, v)$ satisfies Chasles' angle invariance property along $x_{1}$-axis if and only if there exists some real constant $C$ such that

$$
\frac{h q}{k f}=C\left(h^{2} \cos ^{2} v+q^{2} \sin ^{2} v\right), \quad \forall u>0, \forall v \in(0,2 \pi) .
$$


Proof: The tangent plane at every point of the helicoid is spanned by

$$
\begin{gathered}
\sigma_{u}=(0, \cos v, \sin v), \\
\sigma_{v}=(k,-u \sin v, u \cos v) .
\end{gathered}
$$

The normal at every point of the helicoid is the vector $N=(n, m, p)$ satisfying $N \cdot \sigma_{u}=0$ and $N \cdot \sigma_{v}=0$, which yield the linear system:

$$
\begin{gathered}
m h^{2} \cos v+p q^{2} \sin v=0, \\
n k f^{2}-m h^{2} u \sin v+p u q^{2} \cos v=0 .
\end{gathered}
$$

To get the normal direction, take $n=\frac{1}{k}$. Then, solving the system (8) we get:

$$
m=\frac{f^{2} \sin v}{h^{2} u} \quad p=-\frac{f^{2} \cos v}{q^{2} u} .
$$

Therefore the unit length normal is:

$$
N=\frac{1}{\sqrt{\frac{f^{2}}{k^{2}}+\frac{f^{4} \sin ^{2} v}{h^{2} u^{2}}+\frac{f^{4} \cos ^{2} u}{q^{2} u^{2}}}}\left(\frac{1}{k}, \frac{f^{2} \sin v}{h^{2} u},-\frac{f^{2} \cos v}{q^{2} u}\right) .
$$

Note that for $\mathbf{i}=(1,0,0)$ we have $\mathbf{i} \cdot \mathbf{i}=f^{2}$. Denote by $\theta$ the measure of the angle between $N$ and the direction $\mathbf{i}$. Then $\cos \theta=\frac{1}{k} \cdot \frac{1}{\|N\|}$. This yields

$$
\cot \theta=\frac{\frac{1}{k}}{\frac{f^{2}}{u} \sqrt{\frac{\sin ^{2} v}{h^{2}}+\frac{\cos ^{2} v}{q^{2}}}}
$$

The plane passing through the point $(k v, u \cos v, u \sin v)$ perpendicular to $\mathbf{i}=$ $(1,0,0)$ satisfies:

$$
(X-k v, Y-u \cos v, Z-u \sin v) \cdot(1,0,0)=0
$$

which yields $f^{2}(X-k v)=0$. Since $f>0$, the equation is $X=k v$. The distance from the point $(k v, u \cos v, u \sin v)$ to the point $(k v, 0,0)$ is

$$
d=\sqrt{h^{2} u^{2} \cos ^{2} v+q^{2} u^{2} \sin ^{2} v}
$$

Writing the condition $\cot \theta=C \cdot d$, we obtain immediately (7).

The previous theorem opens the path for many examples of such metrics. Take for example $h=q$. Then one gets immediately that any function $h=\frac{\sqrt{2}}{k C f}$ 
provides such an example. This proves that there are infinitely many Riemannian metrics on $\mathbb{R}^{3}$. In a particular case we observe that the classical helicoid preserves Chasles' angle invariance property when isometrically immersed into such such an ambient endowed with a metric satisfying (7).

As a particular case of this analysis, we obtain the following.

Corollary 4.2. Let $\sigma:(0, \infty) \times(0,2 \pi) \rightarrow \mathbb{R}^{3}, \sigma(u, v)=(k v, u \cos v, u \sin v) \subset$ $\mathbb{R}^{3}$, with $k>0$, be an isometric immersion into the three dimensional real space endowed with the warped metric given by

$$
d s^{2}=d x_{1}^{2}+h^{2}\left(x_{1}\right)\left(d x_{2}^{2}+d x_{3}^{2}\right),
$$

with the $h \in C^{\infty}\left(\mathbb{R}^{3}\right), h\left(x_{1}, x_{2}, x_{3}\right)>0$. Then the helicoid given by $\sigma(u, v)$ satisfies Chasles' angle invariance property along $x_{1}$-axis.

Proof: In this setting, $\cot \theta=\frac{u h}{k}$, and $d=u h$, which proves that the proportionality constant is $C=\frac{1}{k}$ (the inverse of the helicoidal pitch) for the case of the warped product metrics.

Example 3. We would like to see an example of such a metric in $\mathbb{R}^{3}$. Take for example the construction inspired from Riemann's original idea of conformal metric:

$$
d s^{2}=d x_{1}^{2}+e^{2 x_{1}}\left(d x_{2}^{2}+d x_{3}^{2}\right) .
$$

This metric provides an example of an ambient space where Chasles' angle invariance property for the helicoid along the $x_{1}$-axis is preserved, in the context of the corollary stated above. Infinitely many metrics with these properties can be constructed.

\section{Is There a Similar Property for the Catenoid? Exten- sion to Rotation Surfaces in the Euclidean Space}

Let $\sigma(u, v)=(x(u, v), y(u, v), z(u, v))$ be a rotation surface along $z$-axis. A natural question is: would there be any natural extension of the angle property we've studied before? More precisely, we are interested in the behaviour of $\angle(N(u, v),(0,0,1))$, which in the most general setting is a function in $u$ and $v$. The formulation of the question that will yield the extension is: find all the tri-twisted metrics that preserve this function (or a certain relation with this function).

We will study this on the catenoid given by the standard parametrization

$$
\sigma(u, v)=\left(c \cosh \frac{v}{c} \cos u, c \cosh \frac{v}{c} \sin u, v\right) .
$$

Then:

$$
\sigma_{u}=\left(-c \cosh \frac{v}{c} \sin u, c \cosh \frac{v}{c} \cos u, 0\right)
$$




$$
\sigma_{v}=\left(\sinh \frac{v}{c} \cos u, \sinh \frac{v}{c} \sin u, 1\right)
$$

We have $\left\|\sigma_{u} \times \sigma_{v}\right\|=c \cosh ^{2} \frac{v}{c}$. The unit normal is:

$$
N(u, v)=\left(\frac{\cos u}{\cosh \frac{v}{c}}, \frac{\sin u}{\cosh \frac{v}{c}},-\tanh \frac{v}{c}\right) .
$$

In consequence, $\cos \angle(N,(0,0,1))=-\tanh \frac{v}{c}$. Note that the distance between an arbitrary point and the $z$ - axis is $d=|v|$. We could interpret $v$ in the previous relation in this way, to compare this property with the helicoid's properties.

In this context, the question we ask is the following. Find all the classes of metrics on $\mathbb{R}^{3}$ of the form

$$
d s^{2}=f^{2}\left(x_{1}, x_{2}, x_{3}\right) d x_{1}^{2}+h^{2}\left(x_{1}, x_{2}, x_{3}\right) d x_{2}^{2}+q^{2}\left(x_{1}, x_{2}, x_{3}\right) d x_{3}^{2}
$$

with the property that $\cos \angle(N,(0,0,1))=-\tanh \frac{v}{c}$. The answer is the following.

Theorem 5.1. Let $\sigma:(0,2 \pi) \times \mathbb{R} \rightarrow \mathbb{R}^{3}, \sigma(u, v)=\left(c \cosh \frac{v}{c} \cos u, c \cosh \frac{v}{c} \sin u, v\right)$ be an isometric immersion into the three dimensional real space endowed with the tri-twisted metric given by

$$
d s^{2}=f^{2}\left(x_{1}, x_{2}, x_{3}\right) d x_{1}^{2}+h^{2}\left(x_{1}, x_{2}, x_{3}\right) d x_{2}^{2}+q^{2}\left(x_{1}, x_{2}, x_{3}\right) d x_{3}^{2},
$$

with the $f, h, q \in C^{\infty}\left(\mathbb{R}^{3}\right), f\left(x_{1}, x_{2}, x_{3}\right)>0, h\left(x_{1}, x_{2}, x_{3}\right)>0$, and $q\left(x_{1}, x_{2}, x_{3}\right)>0$. Then the catenoid given by $\sigma(u, v)$ satisfies $\cos \angle(N,(0,0,1))=-\tanh \frac{v}{c}$ if and only if

$$
q^{4}\left(h^{4}+f^{2} \tan ^{2} u\right)=h^{4} f^{4} \sec ^{2} u\left(q \cosh ^{2} \frac{v}{c}-\sinh ^{2} \frac{v}{c}\right)
$$

Proof: The dot product is given by $f^{2} d x_{1}^{2}+h^{2} d x_{2}^{2}+q^{2} d x_{3}^{2}$. We need to find the unit normal

$$
N=(a(u, v), b(u, v), m(u, v))
$$

satisfying the following conditions:

$$
N \cdot \sigma_{u}=0, \quad N \cdot \sigma_{v}=0
$$

These two conditions yield the system of two equations:

$$
-a \cdot f^{2} \cdot c \cosh \frac{v}{c} \sin u+b \cdot h^{2} \cdot c \cosh \frac{v}{c} \cos u=0,
$$




$$
a \cdot f^{2} \cdot \sinh \frac{v}{c} \cos u+b \cdot h^{2} \cdot \sinh \frac{v}{c} \sin u+m q^{2}=0 .
$$

From equation (17) we get:

$$
b=a \frac{f^{2}}{h^{2}} \tan u .
$$

If we take $a=h^{2}$, we obtain $b=f^{2} \tan u$. Replacing these values in (18) we get:

$$
m=-\frac{h^{2} f^{2}}{q^{2}} \sinh \frac{v}{c} \sec u .
$$

Therefore, the normal should be in the direction of the vector:

$$
\left(h^{2}, f^{2} \tan u,-\frac{h^{2} f^{2}}{q^{2}} \sinh \frac{v}{c} \sec u\right) .
$$

Its squared magnitude is:

$$
h^{4}+f^{4} \tan ^{2} u+\frac{h^{4} f^{4}}{q^{4}} \sinh ^{2} \frac{v}{c} \sec ^{2} u .
$$

The cosine of the angle we study is:

$$
\begin{gathered}
\cos \angle(N,(0,0,1))=\frac{N \cdot(0,0,1)}{\|N\| \cdot 1}= \\
=\frac{m \cdot q^{2}}{q \cdot \sqrt{h^{4}+f^{2} \tan ^{2} u+\frac{h^{4} f^{4}}{q^{4}} \sinh ^{2} \frac{v}{c} \sec ^{2} u}=} \\
=\frac{-h^{2} f^{2} \sinh \frac{v}{c} \sec u}{\frac{q}{q^{2}} \sqrt{q^{4} h^{4}+q^{4} f^{2} \tan ^{2} u+h^{4} f^{4} \sinh ^{2} \frac{v}{c} \sec ^{2} u}}
\end{gathered}
$$

The condition we ask is that this last quantity equals - tanh $\frac{v}{c}$. By setting these two quantities equal and squaring, after simplifications we obtain relation (16).

The previous theorem suggests that a general characterization is possible in the three-dimensional real space as ambient. It is very natural to pursue this extension in $\mathbb{R}$ endowed with the usual dot product. We obtain the following.

Theorem 5.2. Consider the surface of rotation obtained by rotating the curve $\gamma(u)=(f(u), 0, g(u))$ about z-axis. This means $\sigma(u, v)=(f(u) \cos v, f(u) \sin v, g(u))$. Then the cosine of the angle between the normal and the direction of the $z$-axis is equal to the function $\phi(u)$ if and only if

$$
\ln f(u)=\int_{a}^{u} \phi(w)\left\|\gamma^{\prime}(w)\right\|^{2} d w+\ln f(a)
$$


Proof: It is well-known that the normal to the given rotation surface is

$$
N=\frac{\sigma_{u} \times \sigma_{v}}{\left\|\sigma_{u} \times \sigma_{v}\right\|}=\frac{1}{f^{2}\left(\dot{f}^{2}+\dot{g}^{2}\right)}(-f \dot{g} \cos v,-f \dot{g} \sin v, f \dot{f}) .
$$

We have immediately that

$$
\cos \angle(N,(0,0,1))=\frac{\dot{f}}{f\left(\dot{f}^{2}+\dot{g}^{2}\right)} .
$$

Given some continuous function $\phi(u)$, set the equality:

$$
\frac{\dot{f}}{f\left(\dot{f}^{2}+\dot{g}^{2}\right)}=\phi .
$$

This generates the differential equation in $u$ :

$$
\frac{\dot{f}}{f}=\phi\left[\dot{f}^{2}+\dot{g}^{2}\right]
$$

By integrating both sides with respect to $u$, we obtain the claimed result on some interval $(a, b)$, i.e. relation (19).

\section{References}

[1] J. Bernstein and Ch. Breiner - Distortions of the helicoid, Geom. Dedicata $\mathbf{1 3 7}(2008), 143-147$.

[2] D. E. Blair and Th. Koufogiorgios - Ruled surfaces with vanishing second Gaussian curvature, Monat. Math. 113(1992), 177-181.

[3] E. Catalan - Sur les surfaces reglées dont l'aire est un minimum, J. Math. Pures et Appl., 7(1842), 203-212.

[4] B.-Y. Chen - Geometry of submanifolds, M. Dekker, New York, 1973.

[5] B.-Y. Chen - Total mean curvature and submanifolds of finite type, World Scientific, New Jersey, 1984.

[6] B.-Y. Chen - Geometry of submanifolds and its applications, Science University of Tokyo, 1981.

[7] B.-Y. Chen - When does the position vector of a space curve always lie in its rectifying plane?, Amer. Math. Monthly, 110 (2003) No.2, 147-152. 
[8] B.-Y. Chen - Pseudo-Riemannian submanifodls, $\delta$-invariants and Applications, World Scientific, 2011.

[9] C. J. Costa - Example of a complete minimal immersion in $\mathbb{R}^{3}$ of genus one and three embedded ends, Bull. Soc. Bras. Mat., 15(1984), 47-54.

[10] D. Hoffman and W. H. Meeks III - Complete embedded minimal surfaces of finite total curvature, Bull. Amer. Math. Soc. 12(1985), 134-136.

[11] D. Hoffman and W.H. Meeks III - Embedded minimal surfaces of finite topology, Ann. of Math., 131(1990), 1-34.

[12] D. Hilbert - Über den Satz von der Gleichheit der Basiswinkel im gleichschenkligen Dreieck, Proc. London Math. Soc. 35(1902/1903), 50-68.

[13] D. Hilbert and S. Cohn-Vossen - Geometry and the Imagination, translated by P. Nemenyi, AMS Chelsea Publishing, 1999 edition.

[14] K. Kenmotsu - Surfaces with Constant Mean Curvature, Translations of Math. Monographs, vol. 221, American Math. Soc., 2003.

[15] T.-T. Li (editor) - Problems and solutions in mathematics, World Scientific, 1998.

[16] J. B. M. Meusiner - Mémoire sur la courbure des surfaces, Mém. des savans étrangers 10 (read in 1776, print 1785), 477-510.

[17] W. H. Meeks III and M. Weber - Bending the helicoid, Math. Ann. 339(2007), 783-798.

[18] B. O'Neill - Semi-Riemannian geometry with applications to relativity, Academic Press, San Diego, 1983.

[19] V. Pambuccian - Euclidean geometry problems rephrased in terms of midpoints and point-reflections, Elem. Math. 60(2005) 19-24.

[20] R. Ponge and H. Reckziegel - Twisted products in pseudo-Riemannian geometry, Geom. Dedicata, 48(1993), 15-25.

[21] A. Pressley - Elementary differential geometry, second edition, Springer Verlag, 2010.

[22] M. Spivak - A Comprehensive Introduction to Differential Geometry, Third edition, Publish or Perish, 1999.

[23] D. J. Struik - Lectures on Classical Differential Geometry, second edition, reprint of the 1950 edition, Dover, 1988. 
ON CHASLES' PROPERTY OF THE HELICOID IN TRI-TWISTED REAL AMBIENT SPACE

Peter T. HO,

Department of Mathematics,

California State University, Northridge,

18111 Nordhoff Street, Northridge, CA 91330, U.S.A.

Email: trung.ho.511@my.csun.edu

Lucy H. ODOM,

Department of Mathematics,

San Francisco State University,

1600 Holloway Ave, San Francisco, CA 94132, U.S.A.

Email: lodom@mail.sfsu.edu

Bogdan D. SUCEAVĂ,

Department of Mathematics,

California State University at Fullerton,

800 N. State College Blvd., Fullerton, CA, 92834-6850, U.S.A.

Email: bsuceava@fullerton.edu 